\title{
Surface-passivated plasmonic nano-pyramids for bulk heterojunction solar cell photocurrent enhancement $\uparrow$
}

\author{
Alec Kirkeminde, ${ }^{a}$ Markus Retsch, ${ }^{b}$ Qian Wang, ${ }^{c}$ Guowei Xu,${ }^{d}$ Rongqing Hui, ${ }^{c}$ Judy Wu ${ }^{d}$ and Shenqiang Ren ${ }^{* a}$
}

Received 26th March 2012, Accepted 13th May 2012

DOI: $10.1039 / \mathrm{c} 2 \mathrm{nr} 30735 \mathrm{a}$

We report that self-assembled gold (Au) nanopyramid arrays can greatly enhance the photocurrent of narrow bandgap organic solar cells using their plasmonic near-field effect. The plasmonic enhanced power conversion efficiency exhibited up to $200 \%$ increase under the AM 1.5 solar illumination.

Organic thin film photovoltaic devices have attracted enormous attention in recent years due to their low-cost, easy processing, lightweight and flexible properties. ${ }^{1,2}$ For an ideal solar cell to have high power conversion efficiency, adequate light absorption in the photoactive layer is required. To achieve this, increasing the thickness of the photoactive layer is an effective method. However, the increased photoactive layer thickness causes a decrease in the inner electric field, which slows down charge drift and increases the possibility of recombination, and hence reduces charge carrier collection. ${ }^{3}$ Designing an efficient solar cell with high light absorption at a relatively small film thickness is a challenge to be resolved.

Previously, it was found that the absorption and emission properties of photoactive materials can be effectively influenced by the nearby resonant plasmon from metal nanostructures which enhance not only Raman scattering, ${ }^{4}$ fluorescence, ${ }^{5}$ and photochemistry, ${ }^{6}$ but also photovoltaic response. ${ }^{7}$ The plasmonic near-field enhancement or improved coupling to guided modes in metal nanostructures will induce resonant absorption in neighboring organic photoactive materials and thus increase the concentration of excitons. ${ }^{8}$ It was shown by theoretical investigation that optical absorption in thin film organic solar cells was greatly enhanced by up to $50 \%$ under the plasmonic effect of metallic gratings. ${ }^{4}$ Kulkarni et al. ${ }^{4}$ showed that charge carrier generation could be enhanced more than 3 times if a thin film of silver nanoprisms was introduced under the organic photovoltaic materials, which could in principle be used to increase photocurrent in organic thin film solar cells. By blending $\mathrm{Au}$

${ }^{a}$ Department of Chemistry, University of Kansas, Lawrence, KS, USA 66045. E-mail: Shenqiang@ku.edu; Fax: +1 785-864-5396; Tel: +1 785864-2315

${ }^{b}$ Physical Chemistry I, University of Bayreuth, 95440 Bayreuth, Germany 'Department of Electrical, Engineering and Computer Science, University of Kansas, Lawrence, KS, USA 66045

${ }^{d}$ Department of Physics and Astronomy, University of Kansas, Lawrence, KS, USA 66045

$\dagger$ Electronic supplementary information (ESI) available. See DOI: $10.1039 / \mathrm{c} 2 \mathrm{nr} 30735 \mathrm{a}$ nanoparticles with poly(3,4-ethylenedioxythiophene)-poly(styrenesulfonate) (PEDOT:PSS), Yang et al. ${ }^{10}$ showed that plasmonic optical absorption can be used to enhance the photovoltaic performance of tandem structured organic solar cells. However, early studies have not shown the improved energy conversion efficiency in organic solar cells when plasmonic metal nanostructures were embedded into the photoactive layer, although plasmon resonant enhanced optical absorption existed; this is due to the reduced hole mobility caused by disturbed ordering in the polymer phase, or the quenching of excited states in the photoactive polymers. ${ }^{11}$

Compared with spherical nanoparticles, triangular shaped nanoparticles show a strong plasmonic resonance at the tips, from both theory ${ }^{12}$ and experiments. ${ }^{9}$ The plasmonic resonance peak of triangular shaped metal NPs can also be tuned across the entire visible region and even into the near-infrared (NIR), which cannot be easily done with spherical particles. ${ }^{13}$ The well-defined and tunable plasmonic properties of triangular nanoparticles triggered extensive research on preparation and application in solar cells. ${ }^{14-17}$ However, these plasmonic metal nanoparticles usually have a disordered distribution, if the metal NPs were blended with photoactive polymers. ${ }^{18}$ In addition, these nanoparticles have a relatively broad size and shape distribution, and many have missing tips, decreasing their usefulness. In this work, Au plasmonic nanopyramid (NPY) structures, prepared via self-assembled nanosphere lithography, are introduced into a narrow bandgap organic solar cell to examine the effect of tunable plasmonic resonance and surface passivation on light absorption and exciton dissociation. With this setup, the plasmonic near-field created by the Au NPYs under illumination acts as the antenna to influence the resonant optical absorption of the nearby photoactive layer, consisting of a silole-thiophene conjugated polymer (P3) and [6,6]-phenyl-C61-butyric acid methyl ester (PCBM). We specifically selected P3 as the photoactive polymer. This silole-thiophene conjugated polymer features increased absorption at larger wavelengths compared to P3HT due to its narrower bandgap. Whereas the overall performance of this polymer is not as good as P3HT yet, which can be ascribed to the lower charge mobility, it is a good demonstrator for the enhancement effect caused by plasmonic resonators, which are beyond $520 \mathrm{~nm}$ for gold structures. The good match of the plasmon resonance with the absorption spectrum of P3 showcases an upper enhancement limit. The synthesis of the P3 conjugated polymer will be published later. The low mobility of the narrow bandgap conjugated polymer P3 limits the thickness of the photoactive layer (the P3 structure is shown in Fig. S1† of ESI), 
which will influence the absorption and photocurrent of the $\mathrm{P} 3 /$ PCBM system. The plasmonic enhanced photovoltaic performance was then systematically studied by controlling the size and surface capping of the NPYs. The plasmonic organic solar cells with optimum surface capped Au NPYs demonstrate a significant photocurrent enhancement and power conversion efficiency improvement with up to $200 \%$ under the AM 1.5 solar illumination.

The fabrication of plasmonic organic solar cells is schematically described in Fig. 1 (see the detailed solar cell fabrication of ESI $\dagger$ ). The polystyrene (PS) nanospheres with different diameters as templates are fabricated into a single monolayer on an ITO patterned glass substrate. ${ }^{16} \mathrm{~A}$ PS nanosphere single layer assembly is shown in Fig. 1a and e. The PS spheres are tightly coordinated to one another and provide an excellent two-dimensional template for NPYs with sharp tips and edges, enabling enhanced plasmonic resonances. ${ }^{19}$ After $30 \mathrm{~nm}$ Au film evaporation followed by a lift-off process (Fig. 1b), the obtained Au NPY arrays show a uniform size and distribution, which ensure a uniform distribution of the plasmonic field enhancement. The dimensions of Au NPYs are controlled by the size of the templating PS nanospheres, which were characterized using scanning electron microscopy (SEM, Fig. 1f) and atomic force microscopy (AFM, Fig. 1g), respectively. Using different sized PS nanospheres of diameters of $1000 \mathrm{~nm}, 480 \mathrm{~nm}, 360 \mathrm{~nm}$ and $240 \mathrm{~nm}$, respectively, side lengths of the NPYs corresponding to $225 \mathrm{~nm}, 110 \mathrm{~nm}, 80 \mathrm{~nm}$ and $55 \mathrm{~nm}$ could be realized. Furthermore, these NPYs can be readily passivated using surface capping ligands such as thiols of different lengths or compositions. This is of great importance to fully utilize the plasmonic resonance in organic thin film solar cells because charge recombination centers usually form if metal nanoparticles are directly introduced without suitable treatment. ${ }^{20}$ In this work, the Au NPY arrays on the patterned ITO glass substrate were additionally covered with an electron blocking layer of PEDOT:PSS so that the tips of the NPYs were close to the photoactive layer of the organic solar cells, but not in direct contact, leading to concentrated optical electric fields near the junction (Fig. 1h, the protruded tips after the spun-cast PEDOT:PSS layer).

A PEDOT:PSS thin film was then spin-coated onto the Au NPY arrays. The height of $\sim 30 \mathrm{~nm}$ was selected for the NPYs by Au evaporation, which is compatible with the thickness of the PEDOT:PSS anode buffer layer. By controlling the spin-coating
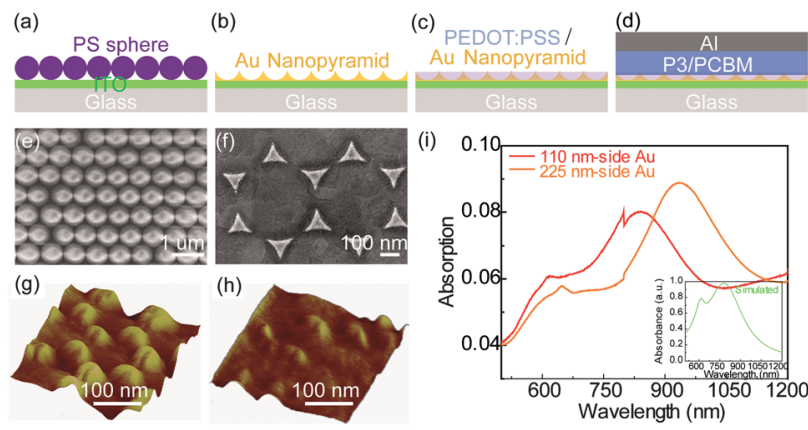

Fig. 1 (a) and (b) Self-assembled PS sphere nanolithography to pattern $\mathrm{Au}$ NPYs. (c) and (d) The PEDOT:PSS layer and complete solar cell structure. (e) and (f) SEM images of PS spheres before Au coating, and Au NPYs after PS removal, respectively. (g) and (h) AFM images of Au NPYs before and after PEDOT:PSS coating, respectively. (i) Absorption spectra of Au NPYs with $110 \mathrm{~nm}$ and $225 \mathrm{~nm}$ side length, the inset image shows a simulated spectrum of $110 \mathrm{~nm}$ side length Au NPYs.

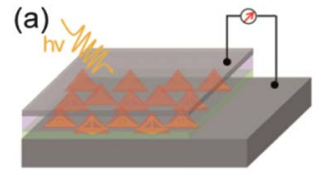

(c)
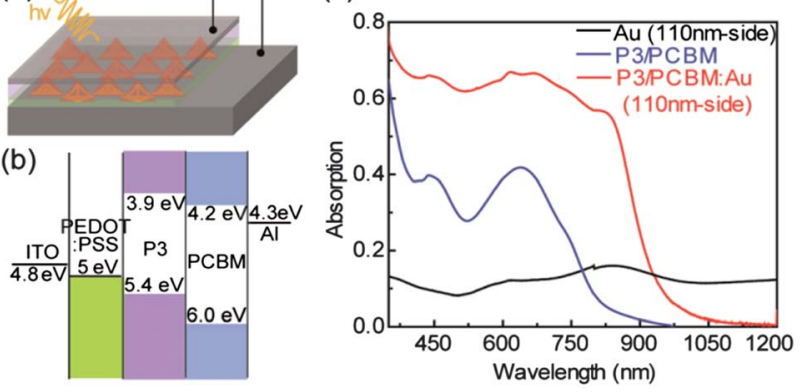

Fig. 2 (a) The schematic image of the Au NPY plasmonic organic solar cell. (b) The flat-band diagram of the plasmonic organic solar cell. (c) Absorption spectra of Au NPYs, the P3/PCBM photoactive layer, and the Au NPY plasmonic enhanced P3/PCBM layer. The Au NPY has a $110 \mathrm{~nm}$ side-length.

process, the Au NPYs are mostly covered by the PEDOT:PSS layer, which provides a smooth surface for optimal contact with the photoactive layer. The AFM surface topology images indicate a protrusion of 2-3 nm peaks on top of the Au NPYs into the photoactive layer. Fig. $1 \mathrm{~g}$ and $\mathrm{h}$ show topographic images of the Au NPYs before and after spin-coating of the PEDOT:PSS layer. The photoactive layer consisted of the conjugated polymer P3 and PCBM $(\sim 70 \mathrm{~nm}$ thickness), which does not show the thickness variation in the presence and in the absence of the Au NPYs. In order to generate a suitable plasmonic effect that influences the above photoactive layer, different Au NPYs with varying side lengths were created and their optical absorption properties were analyzed. Fig. 1i shows that the side lengths indeed play an important role in the plasmonic resonant absorption. The larger side length of Au NPYs leads to a red-shifted plasmonic absorption peak as well as a stronger absorption. An optimum enhancement is expected, when the plasmon resonance of Au NPYs matches the absorption peak of the photoactive P3/PCBM layer.

Fig. 2a shows the schematic diagram of plasmonic organic solar cells discussed in this work. Au NPYs sit on top of an ITO electrode and are coated with PEDOT:PSS, which acts as the anode buffer layer. P3, a p-type narrow bandgap organic conjugated polymer (a more detailed description of the polymers is presented in the ESI + ), is used in conjunction with PCBM to create the type-II heterojunction. Flat-band alignment of the prepared organic solar cell is shown in Fig. 2b. The plasmonic resonance enhanced light absorption of the organic solar cell with the $110 \mathrm{~nm}$ side-length $\mathrm{Au}$ NPYs is shown in Fig. 2c. It was found that a side length of $110 \mathrm{~nm}$ of Au NPYs created with a $480 \mathrm{~nm}$ PS nanosphere generated the optimal plasmonic resonance to effectively couple with the P3/ PCBM photoabsorption from $600 \mathrm{~nm}$ to $900 \mathrm{~nm}$. This cannot be attributed to the absorption of the Au NPYs themselves due to their weak light absorption intensity (Fig. 2c). The plasmonic near-field effect is used to enhance light absorption of organic photoactive materials. ${ }^{21,22}$ In addition, the plasmonic electric field enhancement can be used to dissociate the excitons generated in the photoactive layer and thus improve the photocurrent.

As mentioned before, the plasmon resonance depends on the geometry of the Au NPYs. Therefore, the photovoltaic performance dependence on the side lengths of Au NPYs was studied. Fig. 3a shows the solar cell performance with different sized Au NPYs passivated with the same ligand, a mercapto polyethylene glycol. In 

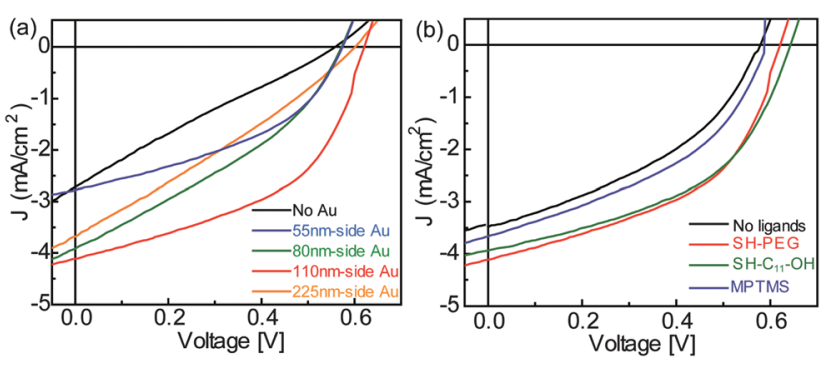

Fig. 3 (a) Current-voltage $(J-V)$ characteristics of solar cells with different side lengths of Au NPYs with SH-PEG ligands. (b) $J-V$ curves of different capping ligands of Au NPYs with $110 \mathrm{~nm}$ side length. The solar cell performance is measured under AM 1.5 solar illumination.

comparison to the reference solar cells (without Au NPYs), the introduction of Au NPYs can improve the photovoltaic performance. Solar cells with Au NPY side-length larger than $55 \mathrm{~nm}$ exhibit a drastic photocurrent enhancement. At a $110 \mathrm{~nm}$ Au side size, the average photocurrent increases (average value \pm standard deviation) from $2.7 \pm 0.2 \mathrm{~mA} \mathrm{~cm}{ }^{-2}$ to $4.1 \pm 0.3 \mathrm{~mA} \mathrm{~cm}^{-2}$ compared to solar cells without plasmonic resonators, which is an increase of $50 \%$. This demonstrates that the plasmonic near-field induced light absorption and electric field enhancement in organic solar cells can be used to effectively improve the photocurrent. The lower reflectivity of the devices with Au NPYs indicates stronger absorption of the incident light (Fig. S2†, ESI). Further increasing the side length of Au NPYs will decrease its photocurrent. Small sized NPYs cannot provide sufficient plasmonic effect, as is demonstrated in Fig. 3a, while Au NPYs with larger size (225 nm side length) feature stronger reflectance, inhibiting light transmission, thus reducing the light absorption of the photoactive layer. The Au NPY showing the best photovoltaic performance, with the side length of $110 \mathrm{~nm}$, that is discussed here is matched with the simulation model of discrete dipole approximation (DDA). ${ }^{19}$ The organic P3/PCBM solar cell with $110 \mathrm{~nm}$ side Au NPY arrays gives an average power conversion efficiency of $1.10 \pm 0.05 \%$ under AM $1.5\left(100 \mathrm{~mW} \mathrm{~cm} \mathrm{~cm}^{-2}\right)$ solar illumination, which is about $200 \%$ more compared to that without Au NPY arrays of $0.36 \pm$ $0.02 \%$ power conversion efficiency. The enhanced exciton dissociation and suppressed recombination due to the protruding plasmonic electric field from Au NPYs are responsible for a better fill factor (FF) of the plasmonic P3/PCBM systems (Fig. 3a). However, the polymer ordering and the series resistance due to Au NPYs could also contribute to the FF difference. The power loss and series resistance are also important for the design of efficient plasmonic enhanced OPV systems. Calculating the various resistive layers has been used to analyze the electrical characteristics of the plasmonic P3/ $\mathrm{PCBM}$ system (Table $\mathrm{S} 1 \uparrow$ of $\mathrm{ESI}$ ). The resistive power loss depends mainly on the P3/PCBM organic photoactive layer, which can be inferred from the series resistance analysis (ESI†). The Au NPYmodified electrode does not contribute to the photocurrent enhancement. To fully utilize the plasmonic effect and to minimize trap spots from Au nanostructures in the photoactive organic layer, we have passivated the Au NPYs by ligands with different lengths and compositions before spin-casting the PEDOT:PSS layer.

It has been shown that the surface of a plasmonic metal should be capped with a thin dielectric layer to prevent exciton quenching through dipole-dipole interactions and charge trapping when metal particles are embedded into photoactive layers. ${ }^{23}$ Dielectric coatings in this work, thiols with different chain lengths and compositions, play an important role in preventing adverse effects of hole traps while offering an extra degree of tunability in spectral response and degree of enhancement of optical absorption. The plasmonic evanescent field of $\mathrm{Au}$ nanoparticles decreases exponentially on moving away from the Au nanoparticle surface. Therefore, we passivated the Au NPYs with $110 \mathrm{~nm}$ side length with different ligands. It was found that the ligand length had an influence on the performance of solar cells. By treating NPYs with different thiol side chains, we measured the photoresponses and found that poly(ethylene glycol) methyl ether thiol (SH-PEG) can have a most effective performance enhancement compared with other ligands such as 11-mercapto-1-undecanol (SH-C11-OH), (3-mercaptopropyl) trimethoxysilane (MPTMS) or no ligands. In comparison, SH-PEG has a longer chain length (about $1.8 \mathrm{~nm}$ ) than $\mathrm{SH}-\mathrm{C} 11-\mathrm{OH}$ (about $1.2 \mathrm{~nm}$ ) and MPTMS (about $0.6 \mathrm{~nm}$ ). One should note that due to the different chemical compositions of these capping agents, additional effects from the different dielectric environments could play an important role in the performance improvement. Considering that all these three molecules may act as insulating barriers, we attribute the different extents of performance improvement to the difference in the ability of inhibiting exciton quenching or charge recombination. Fig. 3b demonstrates that a solar cell using Au NPYs with the shortest capping shell, MPTMS, still has an improved performance compared to that without shell capping. Increasing the capping shell length leads to a more enhanced performance, demonstrating a more efficient exciton quench-inhibiting ability. This is to some extent similar to that researched by Topp et al. ${ }^{11}$ where solar cells could gain a positive effect when dodecylamine, a long insulating molecule, capped Au was used in the hybrid system while a negative effect was obtained in the case of a short molecule, pyridine. In this work, the $\mathrm{Au}$ NPY array was located under the photoactive layer and its plasmonic resonance induced near-field can be more effectively utilized by decreasing the possible phase contact between the Au and the P3/PCBM phase which may cause charge recombination. Thus even a small molecule such as MPTMS can improve solar cell performance although the surface may not be completely capped due to its short and rigid chains. Solar cells with SH-PEG capped NPYs (1.1\% efficiency) show a performance enhancement of about $31 \%$ compared to the one without ligand treatment $(0.84 \%)$.

In order to further understand the plasmonic near-field effect on the photocurrent of solar cells, the external quantum efficiency (EQE) with and without Au NPYs (110 nm side length) was studied and the result is included in Fig. 4a. The EQE spectrum is largely improved using the plasmonic Au : SH-PEG NPYs, compared to the reference cells. The enhanced EQE at $600 \mathrm{~nm}$ wavelength is primarily

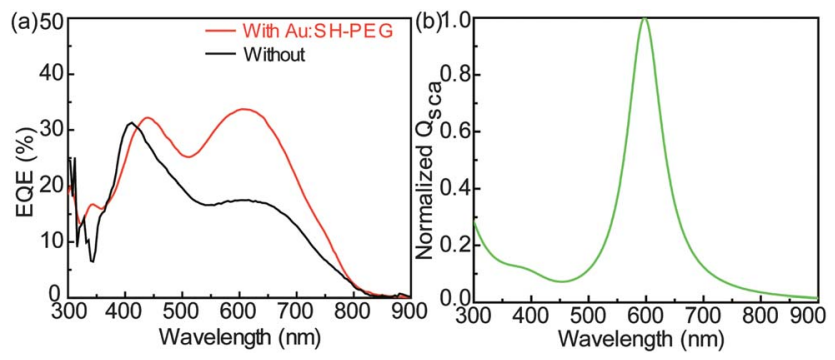

Fig. 4 (a) External quantum efficiency of an organic solar cell with SHPEG capped Au NPYs (red) and without (black). (b) Plasmonic resonance simulation of Au NPYs. 
introduced by the resonant field enhancement near the tips of the $\mathrm{Au}$ NPYs that intrude into the P3/PCBM photoactive layer. Based on the Mie scattering theory and assuming that the particle size is much smaller than the wavelength, ${ }^{24}$ the scattering cross-section can be expressed as,

$$
Q_{\mathrm{sca}}=\frac{A}{\lambda^{4}}\left|\left(\varepsilon_{\mathrm{Au}}-\varepsilon_{\mathrm{P} 3}\right) /\left(\varepsilon_{\mathrm{Au}}+2 \varepsilon_{\mathrm{P} 3}\right)\right|^{2}
$$

where $\lambda$ is the wavelength, $A$ is a proportionality constant which depends on the particle geometric cross-section, and $\varepsilon_{\mathrm{Au}}$ and $\varepsilon_{\mathrm{P} 3}$ are dielectric constants of $\mathrm{Au}$ and $\mathrm{P} 3 / \mathrm{PCBM}$, respectively. Assuming $\varepsilon_{\mathrm{P} 3}$ $=1.9$ and using the Drude-Lorentz model for gold, a normalized scattering cross-section can be calculated as shown in Fig. $4 b{ }^{25}$ This further demonstrates that efficient light absorption and exciton dissociation of the P3/PCBM photoactive layer are strongly correlated with plasmonic resonance field enhancement. The increased free charge carriers contribute to the photocurrent, leading to enhanced power conversion efficiency of organic solar cells. In addition, the reduced charge carrier losses from the geminate recombination in the P3 phase lead to a large built-in electric field and a high $V_{\text {oc }}$ (Fig. 3a and $b$ ). The resulting geminate recombination by increased free charge carriers has been confirmed using the different light intensities (ESI, Fig. S3 $\dagger)^{26-29}$

Plasmonic-mediated absorption enhancement may be originated from scattering and near-field enhancement effect. An additional effect to enhance the solar cell efficiency can originate from increased light scattering due to the presence of scattering objects such as the NPYs. To gauge this contribution, we measured scattering spectra of glass and Au NPY modified glass using an integrating sphere spectrometer. From this, we find that enhanced scattering will play a minor role in our device (details are given in the ESI, Fig. S3†). It is reasonable to infer the absorption enhancement coming from the near-field enhancement which can be described entirely as a plasmonic effect from the Au NPYs. The simulated extinction crosssection spectra of Au NPYs in the PEDOT:PSS and P3/PCBM layer are shown in Fig. 5 using the finite-difference time-domain (FDTD) simulation based on FULLWAVE simulation package18 (ESI $\dagger$ ). Fig. 5a shows the 3D structure of one Au NPY with $110 \mathrm{~nm}$ sidelength. In order to see the surface plasmonic effect, two monitors were placed in the positions shown in Fig. 5a. Since plasmon coupling takes place only in its near-field region, the electric fields aligned in the $x-y$ and $z$ direction are simulated, and the results are shown in Fig. 5b and c, respectively. An enhanced electric field was observed in both the $x-y$ and $z$ directions in these conditions, in particular, with the sharp edges and the thickness of PEDOT:PSS of about $30 \mathrm{~nm}$, which is complementary to the Au NPY thickness $(30 \mathrm{~nm})$. The first monitor measures the simulated $|E|^{2}$ image cutting through the center of NPYs vertically, and the power distribution in the same plane is shown in Fig. 5b, which shows a decay length of $15 \mathrm{~nm}$. The other monitor cuts horizontally through the NPYs and provides the $x-y$ field enhancement map in Fig. 5c. According to Fig. 5b and c, high field intensity occurs at the sharp corners of the Au NPYs and up into the organic photoactive layer.

In this work, we have successfully demonstrated that plasmonic near-field enhancement from Au NPY array structures can be used to engineer optical absorption so as to enhance the photocurrent of organic solar cells. It is shown that the performance of the solar cell can be improved by optimizing the light absorption in the photoactive layer by plasmonic field enhancement, which is controlled by (a)

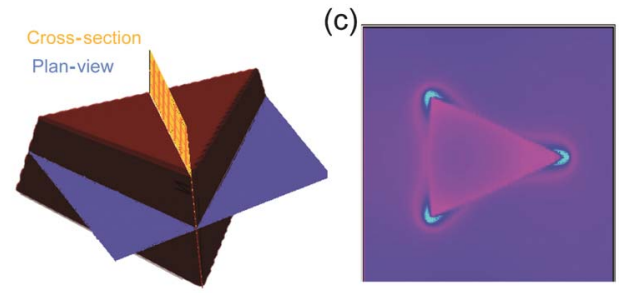

(b)

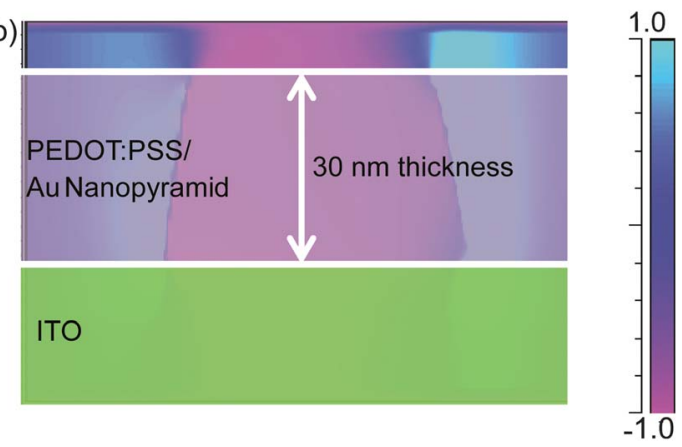

Fig. 5 (a) An example of the structure used in simulation data acquisition. (b) Corresponding simulation data obtained for the cross-section. (c) Simulation data obtained when looking at the plane-view.

the dimension of Au NPYs and their surface capping ligands. Among four different side lengths, Au NPYs with $110 \mathrm{~nm}$ optimal side length show $200 \%$ increase of power conversion efficiency under AM 1.5 $100 \mathrm{~mW} \mathrm{~cm} \mathrm{~cm}^{-2}$ solar illumination compared to solar cells without plasmonic resonators. A power conversion efficiency up to $1.1 \%$ was achieved. The SH-PEG capping ligand of Au NPYs showed further enhancement by about $31 \%$ in comparison with no ligand treatment. Our approach can be applied to a wide range of nanostructured solar cells and is also compatible with conventional solution processing, thereby offering a generic method for the fabrication of highly efficient plasmon enhanced organic solar cells.

\section{Acknowledgements}

S. R. thanks the University of Kansas for its startup and new faculty general research fund (NFGRF) financial supporting and a subcontract from Department of Energy award (DESC0005448). J. W. was supported in part by ARO contract no. ARO-W911NF-09-1-0295 and NSF contract nos. NSF-DMR-0803149, 1105986 and NSF EPSCoR-0903806, and matching support from the State of Kansas through Kansas Technology Enterprise Corporation.

\section{References}

1 J. Li, M. Yan, Y. Xie and Q. Qiao, Energy Environ. Sci., 2011, 4, 4276.

2 G. Yu and A. J. Heeger, J. Appl. Phys., 1995, 78, 4510.

3 C. L. Braun, J. Chem. Phys., 1984, 80, 4157.

4 M. Stavytska-Barba, M. Salvador, A. Kulkarni, D. S. Ginger and A. Kelley, J. Phys. Chem. C, 2010, 42, 20788.

5 A. Kinkhabwala, Z. Yu, S. Fan, Y. Avlasevich, K. Mullen and W. E. Moerner, Nat. Photonics, 2009, 3, 654.

6 K. Watanabe, D. Menzel, N. Nilius and H. Freund, Chem. Rev., 2006, 106, 4301.

7 E. F. Vivian, A. V. Marc, M. Claire van Lare, E. I. S. Ruud, A. A. Harry and P. Albert, Nano Lett., 2011, 11, 4239.

8 J. N. Munday and H. A. Atwater, Nano Lett., 2011, 11, 2195.

9 P. K. Abhishek, M. N. Kevin, M. Keiko, R. G. Samuel and D. S. Ginger, Nano Lett., 2010, 10, 1501. 
10 J. Yang, J. You, C. C. Chen, W. C. Hsu, H. R. Tan, X. W. Zhang, Z. Hong and Y. Yang, ACS Nano, 2011, 5, 6210.

11 K. Topp, H. Borchert, F. Johnen, A. Tunc, M. Knipper, E. Hauff, J. Parisi and K. Al-Shamery, J. Phys. Chem. A, 2010, 114, 3981.

12 K. L. Kelly, E. Coronado, L. L. Zhao and G. C. Schatz, J. Phys. Chem. B, 2003, 107, 668.

13 V. Bastys, I. Pastoriza-Santos, B. Rodríguez-González, R. Vaisnoras and L. M. Liz-Marzán, Adv. Funct. Mater., 2006, 16, 766.

14 I. Santos and L. Liz-Marzán, J. Mater. Chem., 2008, 18, 1724.

15 X. Lu, M. Rycenga, S. E. Skrabalak, B. Wiley and Y. Xia, Annu. Rev. Phys. Chem., 2009, 60, 167.

16 M. Retsch, Z. Zhou, S. Rivera, M. Kappl, X. Zhao, U. Jonas and Q. Li, Macromol. Chem. Phys., 2009, 210, 230.

17 R. Jin, Y. W. Cao, C. A. Mirkin, K. L. Kelly, G. C. Schatz and J. G. Zheng, Science, 2001, 294, 1901.

18 M. Keiko, Y. Chen, F. T. Andreas, P. K. Abhishek, J. P. Ilan, M. M. Andrea and D. S. Ginger, Nano Lett., 2010, 10, 2598.

19 A. M. Angulo, C. Noguez and G. C. Schatz, J. Phys. Chem. Lett., 2011, 2, 1978.
20 J. Wu, F. Chen, Y. Hsiao, F. Chien, P. Chen, C. Kuo, M. H. Huang and C. Hsu, ACS Nano, 2011, 5, 959.

21 H. A. Atwater and A. Polman, Nat. Mater., 2010, 9, 205.

22 J. A. Schuller, E. S. Barnard, W. S. Cai, Y. C. Jun, J. S. White and M. L. Brongersma, Nat. Mater., 2010, 9, 193.

23 J. Y. Lee and P. Peumans, Opt. Express, 2010, 18, 10078.

24 H. C. van de Hulst, Light Scattering by Small Particles, John Wiley \& Sons, New York, 1957.

25 W. Lee, S. Chuang, H. Chen, W. Su and C. Lin, Thin Solid Films, $2010, \mathbf{5 1 8}, 7450$

26 S. Q. Ren, N. Zhao, S. C. Crawford, M. Tambe, V. Bulović and S. Gradečak, Nano Lett., 2011, 11, 408.

27 R. A. Marsh, C. R. McNeill, A. Abrusci, A. R. Campbell and R. H. Friend, Nano Lett., 2008, 8, 1393.

28 C. G. Shuttle, B. O'Regan, A. M. Ballantyne, J. Nelson, D. D. C. Bradley and J. R. Durrant, Phys. Rev. B: Condens. Matter Mater. Phys., 2008, 78, 113201.

29 C. G. Shuttle, A. Maurano, R. Hamilton, B. O'Regan, J. C. de Mello and J. R. Durrant, Appl. Phys. Lett., 2008, 93093311. 